\title{
Small molecules as central nervous system therapeutics: old challenges, new directions, and a philosophic divide
}

\author{
William A Banks ${ }^{*, 1,2}$ \& Nigel H Greig ${ }^{3}$ \\ ${ }^{1}$ Geriatric Research Education \& Clinical Center, Veterans Affairs Puget Sound Health Care System, Seattle, WA, USA \\ ${ }^{2}$ Division of Gerontology \& Geriatric Medicine, Department of Medicine, University of Washington School of Medicine, Seattle, \\ WA, USA \\ ${ }^{3}$ Drug Design \& Development Section, Translational Gerontology Branch, Intramural Research Program, National Institute on \\ Aging, National Institutes of Health, Baltimore, MD, USA \\ *Author for correspondence: wabanks1@uw.edu
}

“ small molecules have been by far the most successful class of CNS therapeutics. On one hand, common problems, such as when CNS-to-blood transporters prevent their accumulation in the brain, challenge their wider development. On the other hand, new approaches to their use as modulators of BBB transport systems open new possibilities"

First draft submitted: 31 August 2018; Accepted for publication: 7 January 2019; Published online: 26 March 2019

Keywords: biologics $\bullet$ blood-brain barrier $\bullet$ brain $\bullet$ central nervous system $\bullet$ clinical trials $\bullet$ pharmacokinetics $\bullet$ small molecules $\bullet$ therapeutics $\bullet$ tight junction proteins $\bullet$ variance

Small molecules are the largest class of therapeutics targeting the central nervous system. Most enter the brain predominantly through the nonsaturable mechanism of transcellular diffusion, although a few, such as L-dopa, use transport systems.

Transcellular diffusion relies on the molecule's ability to pass through the lipid membrane of the target cell. Compounds with a higher molecular weight, more hydrogen bond donors, more hydrogen bond acceptors and low lipid solubility are at a disadvantage for crossing by this route [1], providing the simple principles underpinning the widely used Lipinski rule of 5 (Ro5). This simple rule of thumb provides a rapid way to evaluate whether a compound has sufficient drug-like physicochemical properties to support appropriate pharmacokinetics to predict whether or not it will reach its drug target. Although transcellular diffusion historically has been the most exploited route for the development of CNS therapeutics, there are nonetheless difficulties with its approach, areas that are unclear as to exact mechanism and major misperceptions.

One set of difficulties relates to the optimization of lipid solubility. Whether the target is a neuron, astrocyte, oligodendrocyte or another cell type, the small molecules must not only partition into the lipid environment of blood-brain barrier (BBB) cells, but also have a reasonable solubility in both the aqueous environments of the brain interstitial fluid and plasma to reach their cell target. Based on past successful neurological drugs, a cLogP (computed log octanol/water partition value) that ranges $1-3$ is most favorable for vascular BBB penetration and CNS activity. The structural approach to achieving lipophilicity is also important. For example, cyclization of small peptides can result in highly lipid soluble compounds, however this cyclization also makes them planar, which often results in them acting as substrates for brain-to-blood efflux systems, such as P-glycoprotein (P-gp). This is illustrated by cycloHis-Pro, which is highly lipid soluble but has very low brain penetration. Nonetheless, low doses of cycloHis-Pro have potent CNS effects, such as reversal of ethanol narcosis [2]. For small molecules, the valuable Ro5 derives from evaluating a subset of past compounds (USAN) that had progressed to Phase II clinical studies and beyond prior to 1997. Notably, other studies, for example evaluating marketed CNS drugs and a set of Pfizer CNS drug candidates ([3] and references within it), have cross-validated the Ro5. Such agents were developed toward CNS targets relevant at that time (predominantly aminergic G-protein-coupled receptors, aminergic transporters and ion channels) for which the core characteristics of (likely to be small, lipophilic and possess a basic amine) 


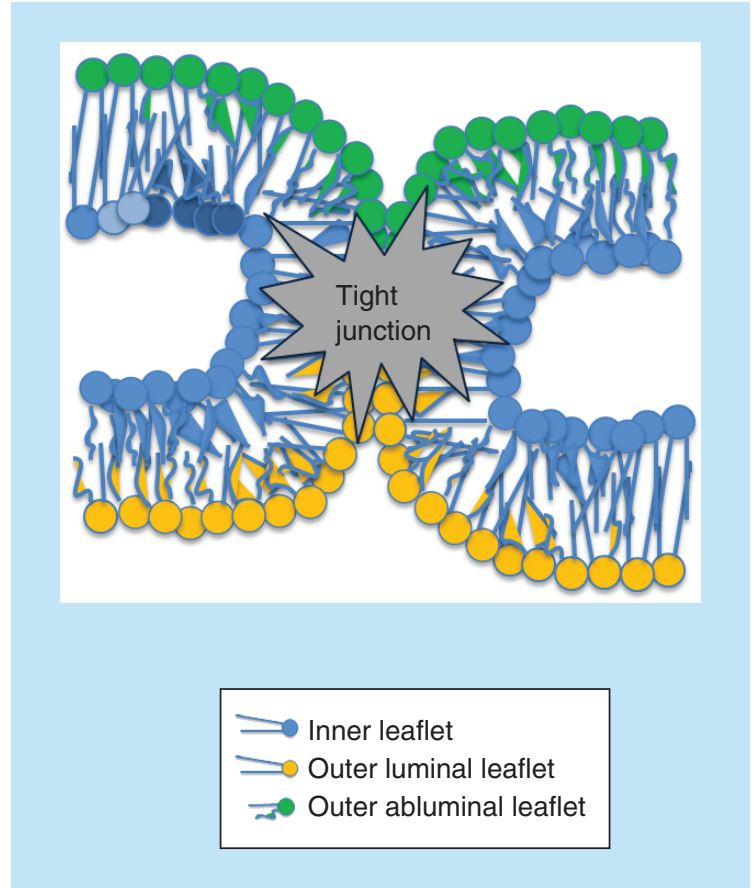

Figure 1. Adjacent brain capillary endothelial cells are joined together by contiguous belts of tight junctions that greatly limit the paracellular flux of solutes. This tight paracellular and transcellular barrier produces a polarized cell with distinct luminal and abluminal membrane compartments such that movement between the blood and the brain can be tightly controlled through regulated cellular transport properties. To view figures in color, please visit: www.futurescience.com/doi/full/10.4155/fmc-2018-0436

may not necessarily match ligand requirements for targets of today and tomorrow (i.e., less traditional intracellular targets: phosphodiesterases, kinases, proteases, etc. - that appear to require larger, more polar ligands [4]). This suggests that chemical space for successful CNS drugs may be slightly larger than previously considered.

The tight junctions of the BBB not only block the passage of substances between cells, but also factor into transcellular diffusion. The tight junctions extend into the outer leaflet of the brain endothelial cell, effectively having a 'fence' function that limits the diffusion of lipids and membrane proteins between the outer leaflets of the luminal and abluminal membranes [5]. Subsequently, two routes have been proposed by which small molecules can cross the BBB by transcellular diffusion. In the first proposed route, the small molecule partitions from the luminal membrane of the barrier cell into the cytoplasm, then into the abluminal membrane and finally into the brain's interstitial fluid. The second proposed route, however, involves the small molecule remaining in the cell membrane but diffusing around the tight junction by moving into the inner leaflet of the cell membrane (Figure 1).

Common practices in the pharmaceutical industry reject substances that are P-gp substrates while promoting those that have a high degree of penetration across the BBB. Both of these practices have historical precedence arguing against them. Morphine, a small molecule which has had widespread clinical use for over a century is a P-gp substrate. In comparison, its diacetylated form, heroin, is not a P-gp substrate and enters the brain rapidly, but is too potent for widespread clinical use. Current industry practice, therefore, would have rejected morphine and forwarded heroin, thus rejecting one of the most clinically prescribed drugs in history while forwarding one with much higher propensity for life-threatening side effects. Occasionally, being a substrate for P-gp can be beneficial as is the case of the widely used anti-histamines cetirizine (Zyrtec), loratadine (Claritin), fexofenadine (Allegra) or desloratadine (Clarinex), and loperamide whose P-gp mediated efflux from brain makes them nonsedating - unlike the first-generation agents epitomized by diphenhydramine (Benadryl).

Many reviews and papers state that there is an absolute molecular weight cut off above which small molecules do not cross the BBB. However, although there is a molecular weight penalty, there is not an absolute cut off. Older literature states the penalty relates to the inverse of the square root of the molecular weight, thus approximately the volumetric radius. The basis for an absolute cut off seems to have originally stemmed from a misinterpretation of a study by Levin [6] and an overinterpretation of the findings of Lipinski. Levin examined the BBB penetration of a series of compounds and found four that essentially did not cross the BBB. These all had molecular weights greater than $400 \mathrm{Da}$, but subsequently these were also discovered to be P-gp substrates. In retrospect, it is being a substrate for a brain-to-blood efflux system, not molecular size, that prevents these compounds from accumulating in the brain. One of Lipinski's Ro5 predicting oral absorption states that a molecular weight under $500 \mathrm{Da}$ 
favors permeability. Subsequent reviews have overinterpreted this rule as if it were absolute. In Lipinski's original publication, $11 \%$ of compounds (over 200 compounds) of the 2245 USAN compounds that had entered Phase II trials had molecular weights greater than $500 \mathrm{Da}$ and $8 \%$ greater than $600 \mathrm{Da}$. In comparison of the 50,427 from which these were drawn, 22\% had MW greater than $500 \mathrm{Da}$ and $14 \%$ greater than 600 Da. Thus, Lipinski concluded that higher molecular weight compounds "are in general less likely to be orally active". In essence, Lipinski and others suggest that small molecule drugs with a lower 'property space' and which comply with the Ro5 have a greater chance of success, not that agents with a larger property space will not be successful CNS drugs. The importance of this concept that there is a molecular weight penalty rather than an absolute cut-off is especially important in the development of 'small molecule biologics' such as the smaller peptides. Lipinski's analysis intentionally eliminated peptides, many of which have MW greater than 400-500 Da and cross the BBB to exert CNS effects, as illustrated by delta sleep-inducing peptide $(849 \mathrm{Da})$ and the opiate peptides and their analogs. CINC1, at a MW of about $7 \mathrm{KDa}$, is the largest known molecule which crosses by transcellular diffusion and exerts a CNS effect [7]. It may be that 'small molecule biologics' have somewhat different rules for lipid solublity, as is the case for anti-parasitic compounds [8]. Lipinski additionally indicated that natural products are an exception to the Ro5 and, interestingly, the four compounds now known to be P-gp substrates from Levin's study are also natural products.

It is interesting to note that particular chemical motifs have appeared to increasingly occur across drug scaffolds, described as 'privileged' structures. Whether these are associated with the presence of pharmacological activity, or with the fact that we are adhering to a common set of rules in relation to drug design - and thus have a preference toward certain chemical skeletons - remains food for thought. It has been noted that 'chemical novelty' does not necessarily increase 'drug discovery' success, as biologically active compounds are not necessarily evenly distributed in chemical space. Thus, the introduction of greater chemical novelty to gain composition of matter patents may drive synthesis away from the 'drug-like' molecule space and so result in higher attrition rate of candidate drugs.

It should be noted that small molecules can also exert effects on the brain indirectly, by pathways which do not require them to cross the $\mathrm{BBB}$. One such mechanism involves their ability to modulate the transport systems for other molecules. In this case, they are likely acting as allosteric regulators of the transport systems. For example, alpha-adrenergics upregulate the rate of leptin transport across the BBB [9]. They also re-induce the transporter for lysosomal enzymes, a transporter that is lost developmentally [10]. Such use has the advantage that the small molecule does not necessarily need to cross the BBB when its site of action is on the luminal surface of the brain endothelial cells. It is also possible that the therapeutic actually entering the brain is endogenously made so that the direct CNS effect is mediated by the patient's own hormone(s).

There is some difficulty in developing small molecules for the treatment of CNS diseases. This originates in part from the failure of clinical trials for drugs which otherwise work well in animal models. In an interesting review article from 2004, Kola and Landis [11] detail the root causes of why compounds undergo attrition during the clinical development stage. Notably, in 1991 the primary cause (accounting for some 40\% of all attrition) was adverse pharmacokinetics/bioavailability; however by 2000 this had fallen to less than $10 \%$ thanks in part to the application of drug design guidelines such as the Ro5. Now, the prime cause of drug failures appears to be lack of efficacy (accounting for some 30\% of attrition) [11,12]. Much of the blame of such failures has been placed on basic science and particularly with the animal models used to predict efficacy in humans. Though animal models reproduce some physiological aspects of human disease, they lack the complexity of the human brain and hence do not predict human response, therefore lacking efficacy. This effect is particularly evident for diseases which have complex etiology, such as neurological or neuropsychiatric disorders.

A much more fundamental problem is the difference in the approach at a near philosophical level between basic research and clinical trials research. This difference in approach extends all the way to how simple statistical results are presented: basic science experiments are often dealing with the elucidation of principles; results are typically presented as the mean with standard error in bar graphs. However, clinical research is often interested in the consistency of an effect across a population, therefore scattergrams are often shown and the standard deviation favored. Thus, the analysis, presentation and interpretation of the simplest comparison is fundamentally different.

It is likely that the decreasing number of investigators who carry out both clinical and basic research have accelerated the rift between the two fields. This decreasing number has likely been accelerated by the increased regulatory burden that accompanies both basic and clinical research, especially when the research involves animal and human subjects. It is very difficult for one individual to have the time and fortitude to deal with the complexity of two very different systems of regulation and oversight. Investigators naturally tend to specialize in navigating the 
administrative burden of their area of research. As a result, clinical and basic research have developed very different approaches to some fundamental problems with few people expert enough in both areas to note the technical drifts. For example, basic research emphasizes altering only one variable at a time and strives to use animals that are as close to one another as possible (genetics, age, etc.). Statistical power is increased by reducing variance. In clinical research, there is an increased emphasis on selecting heterogeneous populations, and statistical power is increased by increasing the population size. However, it should be noted that methods used to increase patient enrollment may result in an increase in variance. For example, including multiple study sites presents the challenge of performing consistent evaluations of variables and outcome measures across those sites [13]. Additionally, drug kinetics, dosing issues and drug levels at the target site can be considered to a nearly obsessive level in a basic science study, only to be simplified to the point of irrelevance in a clinical trial [14]. In basic science research, drug development is invariably hypothesis and mechanism driven; in clinical development, drug-target engagement may be evaluated early but is too often replaced by efficacy versus no efficacy outcome measures focused toward gaining FDA new drug application (NDA) approval - this provides limited insight into precisely why so many drugs fail. Even drug development programs which are halted can be seen as 'good failures' when the termination has convincingly tested a proposed therapeutic mechanism grounded on a soundly based scientific hypothesis, or provides a clear route for future studies. As a final example, in basic science research, an experimental animal not receiving the experimental treatment would be excluded from analysis, whereas in clinical research a subject not adhering to protocol or even ingesting the test drug would be kept in the study under the philosophy of intention to treat.

One tactic toward reconciling the translational divide could involve basic research and clinical research adopting an exchange of approaches. For example, a rodent study could, after the initial study, have a follow-up in which other strains of rodents of varying ages are combined. Even if statistical significance is lost, it would be important to note that such variables introduce an extra level of variance. Initial, smaller clinical trials could focus on a homogenous population most likely to benefit from the treatment and small enough to monitor treatment exposure and other relevant variables, later introducing factors that increase variance. Reviews of clinical trials, rather than implementing such heavy reliance on meta-analysis, could consider the differences among those trials which might account for why some found a therapeutic effect and others did not, thus possibly discovering important parameters in determining therapeutic potency. This reverse-translational approach could provide insight into future clinical trials or identify subpopulations of patients who would benefit from the medication, instead of denying all patients access to the drug because it failed in a large clinical trial with a diverse patient population. As a bottom line, it is always important to evaluate how clinical trial conditions and results relate to the 'real world' to provide appropriate and unbiased clinical trial-evidenced guidance to practitioners $[15,16]$.

A further major cause of drug attrition during clinical development relates to toxicity (accounting for some 30\% of drug failures [11]). Interestingly, the physicochemical drug properties which best associate with in vivo toxicity appear to be a high lipophilicity and a low polarity [17]. A $C \operatorname{logP}>3$ and a topological polar surface area $<75$ $\AA^{2}$ is associated with a 6:1 increased risk for development of an adverse event [17], likely due to the promiscuity of increasingly lipophilic compounds [18]. Hence, the approach of improving the CNS penetrability of a drug by increasing its lipophilicity and reducing its polarity can drive a compound toward a higher risk for toxicity [3]; thereby, generating a Rubik's cube conundrum when trying to dial in the optimal properties for drug actions and safety.

In summary, small molecules have been by far the most successful class of CNS therapeutics. On one hand, common problems, such as when CNS-to-blood transporters prevent their accumulation in the brain, challenge their wider development. On the other hand, new approaches to their use as modulators of BBB transport systems open new possibilities. Their development is also hindered by the difficulties in translational medicine, which we think is mainly due to the difference in the approaches that have arisen between basic science and clinical trials, as to the strength of the science itself. It is clear that the environment changes as one moves through each of the stages of the drug development process - from the initial conception of the drug and its intended target actions to the later evaluation of the drug in the human disease condition. A challenging diversity of complexities continuously emerges during the drug development process. Knowing the nature of these issues and how they may complicate the path to success allows for the flexibility to potentially offset them. This could be achieved by both the application of predictive tools and preclinical-clinical cross talk to guide decision-making and address the high attrition rates in CNS drug development. 


\section{Financial \& competing interests disclosure}

This work was supported in part by the Intramural Research Program, National Institute on Aging, NIH. The authors have no other relevant affiliations or financial involvement with any organization or entity with a financial interest in or financial conflict with the subject matter or materials discussed in the manuscript apart from those disclosed.

No writing assistance was utilized in the production of this manuscript.

\section{References}

1. Lipinski CA, Lombardo F, Dominy BW, Feeney PJ. Experimental and computational approaches to estimate solubility and permeability in drug discovery and developmental settings. Adv. Drug Deliv. Revs. 23(1-3), 3-25 (1997).

2. Banks WA, Kastin AJ, Akerstrom V, Jaspan JB. Radioactively iodinated cyclo(His-Pro) crosses the blood-brain barrier and reverses ethanol-induced narcosis. Am. J. Physiol. 264(5 Pt. 1), E723-E729 (1993).

3. Wager TT, Chandrasekaran RY, Hou X et al. Defining desirable central nervous system durg space through the alignment of molecular properties, in vitro ADME, and safety attributes. ACS Chem. Neurosci. 1(6), 420-434 (2010).

4. Rankovic Z. CNS physicochemical property space shaped by a diverse set of molecules with experimentally determined exposure in hte mouse brain. J. Med. Chem. 60(14), 5943-5954 (2017).

5. van Meer G, Simons K. The function of tight junctions in maintaining differences in lipid composition between the apical and the basolateral surface domains of MDCK cells. EMBO J. 5(7), 1455-1464 (1986).

6. Levin VA. Relationship of octanol/water partition coefficient and molecular weight to rat brain capillary permeability. J. Med. Chem. 23(6), 682-684 (1980).

7. Pan W, Kastin AJ. Changing the chemokine gradient: CINC1 crosses the blood-brain barrier. J. Neuroimmunol.115(1-2), 64-70 (2001).

8. McKerrow JH, Lipinski CA. The rule of five should not impede anti-parasitic drug development. Int. J. Parasitol. Drugs Drug Resist. 7(2), 248-249 (2017).

9. Banks WA. Enhanced leptin transport across the blood-brain barrier by alpha1-adrenergic agents. Brain Res. 899(1-2), 209-217 (2001).

10. Urayama A, Dohgu S, Robinson SM, Sly WS, Grubb JH, Banks WA. Alpha adrenergic induction of transport of lysosomal enzyme across the blood-brain barrier. PLoS ONE 10(11), e0142347 (2015).

11. Kola I, Landis J. Can the pharmaceutical industry reduce attrition rates? Nat. Rev. Drug Discov. 3(8), 711-715 (2004).

12. Waring MJ, Arrowsmith J, Leach AR et al. An analysis of the attrition of drug candidates from four major pharmaceutical companies. Nat. Rev. Drug Discov. 14(7), 475-486 (2015).

13. Becker RE, Greig NH. Lost in translation: neuropsychiatric drug development. Sci. Transl. Med. 2(61), 61 rv66 (2010).

14. Becker RE, Greig NH, Giacobini E. Why do so many drugs for Alzheimer's disease fail in development? Time for new methods and new practices? J. Alzheimer's Dis. 15(2), 303-325 (2008).

15. Becker RE, Greig NH. Neuropsychiatric clinical trials: should they accommodate real-world practices or set standards for clinical practices? J. Clin. Biochem Nutr. 29(1), 56-64 (2009).

16. Becker RE, Seeman MV, Greig NH, Lahiri DK. What can triumphs and tribulations from drug research in Alzheimer's disease tell us about the development of pscyhotropic drugs in general? Lancet Psychiatry 2(8), 756-764 (2015).

17. Hughes JD, Blagg J, Price DA et al. Physicochemical drug properties associated with in vivo toxicological outcomes. Biorg. Med. Chem. Lett. 18(8), 4872-4875 (2008).

18. Leeson PD, Springthorpe B. The influence of drug-like concepts on decision-making in medicinal chemistry. Nat. Rev. Drug Discov. 6(11), 881-890 (2007). 
